\title{
La terminología de la gastronomía puertorriqueña: reflexiones en torno a su trasfondo y representación
}

\author{
The Terminology of Puerto Rican Gastronomy: Reflections \\ on its Background and Representation
}

\author{
Ismarie DÍAZ FLORES \\ Universidad de Salamanca / Doctora en Traducción y Mediación Intercultural \\ ismarie@usal.es
}

Recibido: julio 2018. Revisado: septiembre 2018. Aceptado: octubre 2018.

Resumen: Nuestro estudio da cuenta del entramado que subyace a la terminología utilizada en cinco libros de recetas de Puerto Rico, publicados en momentos clave de la historia puertorriqueña: la década de los cincuenta del siglo XX, marcada por los cambios políticos en la isla, y la primera década del siglo XXI. Por medio del análisis de la terminología, a la luz de múltiples niveles de contexto, entre los que se incluye el contexto cultural, el dominio, la producción textual y el uso de los términos en el corpus, llegamos a conclusiones respecto a la conceptualización del dominio culinario en Puerto Rico. A partir de estos hallazgos, proponemos una base de datos terminológica que considera los aspectos culturalmente marcados de esta terminología. A fin de ilustrar la forma en que estos aspectos se integran en la investigación y en la forma en que se representa el conocimiento especializado en la base de datos, describimos, a modo de ejemplo, cómo inciden estos contextos en la configuración y representación de los términos que forman parte de la categoría de ingredientes denominados viandas.

Ismarie DÍAZ FLORES

La terminología de la gastronomía puertorriqueña: reflexiones en torno a su trasfondo y representación 
Palabras clave: terminología; traducción; gastronomía; cocina; cultura.

\begin{abstract}
Our investigation looks into the background behind the terminology used in five Puerto Rican cookbooks, published in two key periods in Puerto Rico's history: the fifties, a period characterized by political changes in the island, and the first decade of the 21 st century. Based on a terminological analysis that takes into account different levels of context, including the cultural context, the culinary domain, the textual production, and the use of the terms in the corpus, we arrive at some/several conclusions regarding the conceptualization of the culinary domain in Puerto Rico. Finally, we propose a terminological database that considers the culture-specific aspects of this terminology. In order to illustrate the various levels of context and how they are incorporated in our research, as well as how the information is represented in the database, we describe how these levels of context influence the configuration and representation of the terms in the ingredient category identified as viandas.
\end{abstract}

Key words: translation; terminology; cuisine; culture.

\title{
1. INTRODUCCIÓN
}

\subsection{Terminología y cultura en la cocina}

La traducción de textos culinarios requiere trabajar con terminología que puede representar todo un reto para el traductor. Ello se debe a la forma en que esta terminología se relaciona con la cultura. Actualmente, estudiosos adscritos a diversas teorías en torno a la Terminología reconocen que la cultura está íntimamente ligada a la comunicación y la representación del conocimiento especializado, dado que condiciona la creación y la adquisición de nuevos conceptos (Lara 1999, Diki-Kidiri 2008, Faber 2012). Percibimos y experimentamos el mundo en el contexto de una cultura: «Cognition is culture-dependent as well, since our modalities of experience and our perception cannot be separated from the environment where we live and our previously stored experiences" (Faber y León Araúz 2014, 138). Dada la relación entre la cognición y la cultura, la terminología de un dominio revela características del entorno cultural en la que se utiliza:

Los términos además de denominar los objetos sirven para expresar las diferencias conceptuales ligadas a la cultura de una comunidad, grupo social o escuela profesional, para preservar dicha cultura más allá de la uniformidad que pretende imponer la globalización informativa actual [...].(Cabré 2005).

En este artículo, nos proponemos explorar cómo la cultura incide en la categorización y en el uso de la terminología en el dominio culinario en el contexto de Puerto

Ismarie DIÁA FLORES

La terminología de la gastronomía puertorriqueña: reflexiones en torno a su trasfondo y representación 
Rico, a partir de nuestros hallazgos como parte de la investigación realizada en Díaz Flores (2017). En la primera parte, describimos las relaciones que se establecen entre los diversos niveles de contexto en los que se inserta la terminología estudiada. Posteriormente, desglosamos los métodos y materiales utilizados en el estudio. De una parte, ofrecemos algunos datos sobre el proceso utilizado para extraer las categorías conceptuales del dominio por medio de recursos como lenguajes de marcado, ontologías sobre cocina y el propio corpus. De otra parte, describimos el corpus utilizado en el estudio. En la tercera parte del artículo, a fin de ilustrar la relación entre el contexto y la terminología estudiada, nos concentramos en el análisis de un conjunto de términos.

En concreto, nos centramos en los términos que forman parte de la categoría viandas. Seleccionamos estos términos dado que esta categoría resulta idónea para ejemplificar la relación entre el contexto histórico, la producción textual y el uso de los términos en la configuración de categorizaciones dentro de un sistema conceptual. Asimismo, este ejemplo ilustra cómo, al hablar de la relación entre terminología y cultura no nos referimos solo a términos aislados que no tienen equivalentes en otras lenguas o culturas, sino, además, a la especificidad cultural de determinados aspectos de un sistema conceptual. Finalmente, presentamos nuestras conclusiones a partir del análisis y la representación en una base de datos de estas unidades terminológicas, a la luz de los diversos niveles de contexto estudiados.

\section{LA TERMINOLOGÍA CULINARIA EN EL MARCO DEL CONTEXTO CULTURAL}

La alimentación es «una acción voluntaria y consciente que consiste en proporcionar al cuerpo esa serie de productos nutritivos que, contenidos en los alimentos, son necesarios para la nutrición» (Martínez Monzó y García Segovia 2015, 75). De estudios en torno a la creación de ontologías y bases de datos relacionadas con la alimentación (Kamel Boulos et al. 2015, Ireland y Møller 2000, Pennington et al. 1994, Stewart Truswell et al. 1991), se desprende que, incluso desde una perspectiva centrada solo en la alimentación, es preciso considerar factores culturales. Al describir las discusiones de los comités de terminológicos de INFOODS, Truswell et al. comentan que los miembros eran conscientes de que los grupos alimentarios eran conceptos que dependían de la cultura. El objetivo de utilizar un sistema basado en facetas era dar cuenta de las características de los alimentos, incluidas aquellas ligadas a la cultura, de manera independiente $(1991,20)$.

Asimismo, al describir su trabajo en el proyecto Words for Food, investigación que se concentraba en la terminología alimentaria desde una perspectiva nutricional, Garzone (2017) observa el papel de los factores culturales en la clasificación. En dicho proyecto, el análisis de tres corpus comparables permitió identificar correspondencias

Ismarie DÍAZ FLORES

La terminología de la gastronomía puertorriqueña: reflexiones en torno a su trasfondo y representación 
entre las lenguas de trabajo. Sin embargo, en muchos casos, la investigación develó profundas diferencias de categorización de los conceptos entre las culturas involucradas. Garzone atribuye estas diferencias, por un lado, a las condiciones de vida de las comunidades estudiadas, lo que incluye su historia, territorio, clima, organización social, tecnología y capacidad de conservación de los alimentos; y, por otro lado, al valor simbólico atribuido a los alimentos y a los rituales asociados con estos (Ibíd, 215).

Cuando trabajamos con la cocina como dominio, los aspectos culturales adquieren más relevancia, pues no nos referimos solo al proceso biológico, sino al «conjunto de platos y reglas" (Montanari 2004, 80) que se desarrollan en torno a dicho proceso. No obstante, como señalan otros expertos en el tema, el concepto de cocina va más allá de un conjunto de ingredientes, técnicas y platos:

Cada cultura posee una cocina específica que implica clasificaciones, taxonomías particulares y un conjunto complejo de reglas que atienden no solo a la preparación y combinación de alimentos, sino también a su cosecha y a su consumo (Fischler 1995, 34).

Por otro lado, el concepto de cocina tiende a asociarse con un grupo humano, como, por ejemplo, una nación, una región o una clase social. En este sentido, se considera un factor clave en la definición de la identidad. Como señala Fischler (1995, 61-87) cuando plantea la paradoja del omnívoro, el ser humano, dado que está capacitado para incorporar a su dieta toda clase de alimentos, debe decidir cuáles comerá y cuáles no. La cocina funciona como respuesta a esa paradoja y permite el desarrollo de una relación de identidad y pertenencia (Ibíd., 68).

La formación de una cocina es un proceso en el que intervienen factores que incluyen la disponibilidad de alimentos y tecnologías, los intercambios culturales y la producción textual (Appadurai 1988, Higman 1998, Fischler 1995, Montanari 2004, Fernández-Armesto 2004, Bak-Geller Corona 2013). Dado que dichos factores inciden en la formación de la cocina y, en consecuencia, en los términos que representan y comunican los conocimientos especializados culinarios, estos elementos deben incorporarse en la documentación y análisis de esta terminología.

En esta línea, al aplicar la teoría basada en marcos al ámbito de la cocina, Faber y Vidal Claramonte (2017) destacan que la microteoría pragmática de dicho acercamiento teórico es una teoría de contextos. Recalcan que cada término se inserta dentro de múltiples niveles de contexto: las palabras ubicadas antes y después del término, la oración, el párrafo, un conjunto de textos y, finalmente, el contexto más amplio de la cultura (Ibíd., 159). Según explican, estos contextos permiten determinar qué información pragmática se incluirá en la entrada terminológica correspondiente a cada término en la base de datos. Este acercamiento es cónsono con el que utilizamos en Díaz Flores (2017), donde desglosamos los múltiples niveles de contexto que rodean la terminología de la gastronomía puertorriqueña: el contexto cultural, la producción textual

Ismarie DIÁZ FLORES

La terminología de la gastronomía puertorriqueña: reflexiones en torno a su trasfondo y representación 
y el uso de los términos en un corpus de recetas de Puerto Rico. A continuación, describimos brevemente los niveles de contexto en los que se inserta la terminología de la gastronomía puertorriqueña.

\subsection{La cocina en el contexto histórico de Puerto Rico}

Dado que nuestro estudio se centra en la gastronomía puertorriqueña, es preciso que ofrezcamos algunos datos sobre el contexto histórico de Puerto Rico. Para efectos de este artículo, nos limitaremos a los datos más pertinentes para la formación de la cocina puertorriqueña, los procesos de categorización inherentes a esta y la representación de su terminología en los textos culinarios.

De las culturas que habitaron la isla antes del período de la Conquista, la más conocida es la última, la taína, dado que se han obtenido datos sobre esta no solo gracias a excavaciones, sino también a fuentes documentales producidas por los cronistas españoles. Picó (2008) afirma que, aunque los datos que nos proveen estas fuentes son parciales, se ha conseguido identificar rasgos sobresalientes de la cultura taína, como datos sobre las prácticas agrícolas y alimentarias. Entre las plantas cultivadas por este grupo se encontraban la yuca, el maíz, el achiote, el tabaco, el ají, frutas y hierbas medicinales.

Tras el arribo de Cristóbal Colón en 1493, como parte de su segundo viaje a América, la isla pasó a formar parte de los dominios españoles. Sin embargo, no fue sino hasta 1508 que se fundó el primer asentamiento español. Tras el descubrimiento de yacimientos de oro, los españoles sometieron a los taínos a trabajos forzados en la minería y la agricultura. Si bien desde 1511 los indígenas se rebelaron en múltiples ocasiones, como consecuencia de las enfermedades y los trabajos forzados, para 1530 la población taína había disminuido grandemente. Hacia esta fecha comenzaron a traerse esclavos africanos. Dado que tiempo después la minería llegó a su fin, la actividad económica se concentró en la agricultura y la ganadería.

En Puerto Rico en la olla, ¿somos aún lo que comimos? (2006), Miguel Ortiz Cuadra plantea cómo los intercambios, las carencias, las expectativas y las luchas sociales entre estos y otros grupos que convivieron en la isla, a lo largo de varios siglos, dieron lugar a que determinados alimentos se convirtieran en los componentes clave de la dieta puertorriqueña: el arroz, las habichuelas, la harina de maíz, el bacalao, las viandas y la carne. Ortiz Cuadra atribuye la concepción actual de la cocina en Puerto Rico a tres factores: la interacción de los conocimientos culinarios de los indígenas con aquellos de los inmigrantes que deseaban reproducir sus memorias culinarias en la isla, el establecimiento de sistemas de alimentación para dar de comer a los esclavos africanos y a los militares que llegaron a la isla entre los siglos XVII y XIX y el deseo de supervivencia en un contexto marcado por la rutina (Ibíd: 317).

Ismarie DÍAZ FLORES

La terminología de la gastronomía puertorriqueña: reflexiones en torno a su trasfondo y representación 
En 1898, como consecuencia de la Guerra Hispanoamericana, Puerto Rico fue otorgado como botín de guerra a los Estados Unidos. Si bien en este punto ya se habían establecido los principales componentes de la dieta, la relación colonial entre Puerto Rico y los Estados Unidos también ha tenido consecuencias en la dieta y en la forma en que se entiende la cocina nacional en la isla. Nos concentraremos en una serie de procesos histórico-sociales que tuvieron lugar entre las décadas del cuarenta y cincuenta, por motivo de su relación con las publicaciones de tema culinario que aparecen en los cincuenta.

Aunque la Ley Jones de 1917 concedió la ciudadanía estadounidense a las personas nacidas en Puerto Rico, no les otorgó todos los derechos que tenían los estadounidenses. No fue sino hasta 1946 que el presidente estadounidense Harry S Truman nombró al primer gobernador de origen puertorriqueño de la isla. En 1947, el Congreso de los Estados Unidos aprobó una enmienda a la Ley Jones, como resultado de la cual, en 1948, por primera vez, los puertorriqueños eligieron por votación a un gobernador puertorriqueño. En julio de 1950, se aprobó la Ley 600, que autorizó una convención para la organización de un gobierno constitucional. En este mismo año, grupos que favorecían la independencia de la isla se alzaron en armas en varios pueblos de la isla y se efectuó un atentado en la residencia del presidente de los Estados Unidos, como parte de lo que se conoció como la Revolución nacionalista. En 1952, se ratificó la constitución del Estado Libre Asociado.

De acuerdo con Duany $(2002,123)$, en la década de los cincuenta emerge en Puerto Rico el nacionalismo cultural -noción que suele oponerse a la de nacionalismo político-, que se caracteriza por el énfasis que se da a aspectos como la historia, la cultura y la lengua. Según explica Duany, los nacionalistas culturales validan su autonomía espiritual por medio de la educación, la recuperación de las tradiciones y otros rituales simbólicos. Duany lo interpreta como una estrategia para reafirmar la identidad puertorriqueña en el contexto de la relación de dependencia política y económica con los Estados Unidos. Esta noción resulta relevante para nuestro estudio ya que podemos interpretar la aparición de libros de recetas puertorriqueños como manifestaciones de este nacionalismo.

\subsection{El rol de la producción textual}

La producción textual juega un papel clave en la formación de la cocina nacional y en la estructuración de categorías culinarias. Aunque actualmente existen numerosos tipos de textos en los que se comunica el conocimiento culinario, en nuestra investigación nos centramos en la terminología utilizada en recetas que forman parte de libros de recetas. La selección de este género se basa en varias consideraciones entre las que se destacan la presencia de descripciones de aspectos técnicos, el grado de

Ismarie DIAZ FLORES

La terminología de la gastronomía puertorriqueña: reflexiones en torno a su trasfondo y representación
CLINA

vol. 4-2, December 2018, 181-205

elSSN: 2444-1961

Ediciones Universidad de Salamanca - CC BY-NC-ND 
especialización y el rol de estos textos en la codificación y validación de determinados valores culinarios.

De acuerdo con Appadurai (1998, 3), la publicación de un libro de recetas representa un interés por normalizar el régimen culinario, transmitir el conocimiento culinario y difundir determinadas tradiciones. Estas publicaciones representan un momento clave en la definición de una cocina nacional:

In any attempt to understand the culture-history of food, the prescriptive texts hold an important place [...]. It can be argued that the emergence of the cookbook marks a critical point in the development of any cuisine and that the specialization and ramification of texts has much to tell about the character of national, regional, and ethnic identities (Higman 1998, 77).

Dicho esto, no debemos caer en el error de pensar que estos textos «crean» la cocina nacional o que representan su totalidad. En la medida que estos textos reclaman la autenticidad y el vínculo de determinados platos con la identidad nacional, excluyen otros tantos (Floyd 2010, Ayora Díaz 2017).

\section{MÉTODOS Y MATERIALES}

\subsection{Corpus}

Los primeros libros de recetas redactados por personas nacidas en la isla aparecieron en la década del cincuenta1. Estos textos fueron Cocine a gusto, de Berta Cabanillas, Carmen Ginorio y Carmen Quirós de Mercado, de 1950, y Cocina criolla, de Carmen Aboy de Valldejuli, de 1954. Cocine a gusto se tradujo como Puerto Rican Dishes, en 1956, bajo la autoría de Berta Cabanillas y Carmen Ginorio, ya que, para entonces, Carmen Quirós de Mercado había fallecido. La editorial Doubleday \& Company publicó la traducción de Cocina criolla en 1963, bajo el título The Art of Caribbean Cookery. En el 1975, Pelican Publishing publicó una versión ampliada titulada Puerto Rican Cookery.

1. El primer libro de recetas de Puerto Rico del que se tiene conocimiento fue el Manual del cocinero puertorriqueño, de 1859. Sin embargo, el autor se identificaba a sí mismo como español y, de acuerdo con Barradas (2010), este libro es una copia de Manual del cocinero cubano, de 1856, con algunas adaptaciones que responden a la variante de la lengua en ambas islas. Asimismo, tenemos noticia de otros libros de recetas puertorriqueñas redactados por autoras de origen estadounidense.

Ismarie DÍAZ FLORES

La terminología de la gastronomía puertorriqueña: reflexiones en torno a su trasfondo y representación 
Las autoras de Cocine a gusto eran profesoras del Departamento de Economía Doméstica de la Universidad de Puerto Rico. Sus conocimientos sobre la composición y las propiedades de los alimentos se plasman en los múltiples textos sobre alimentación que acompañan las recetas. Berta Cabanillas, por su parte, publicó los trabajos de investigación El puertorriqueño y su alimentación a través de su historia (Siglos XVI-XIX) (1973) y El folklore en la alimentación puertorriqueña (1983). Por su parte, Valldejuli, autora de Cocina criolla, no contaba con preparación formal sobre cocina y no pretendía acercarse al tema desde una perspectiva especializada.

Si bien los textos difieren en lo concerniente al nivel de especialización de las autoras, se asemejan en su propósito didáctico y en el interés que muestran por proveer de una definición de la «cocina puertorriqueña». A modo de ejemplo, en la introducción de la versión en español de Cocine a gusto, las autoras afirman:

Un sentimiento patriótico nos inspira a publicar este libro: darle forma permanente a las recetas que han ido pasando de boca en boca a través de generaciones, para ayudar a la consagración de la cocina regional y ofrecer a nuestro pueblo una obra en el vernáculo (Cabanillas et al. [1950] 1983, 17).

Tras la publicación de estos primeros libros de recetas, a lo largo de los años, aparecieron otros recetarios en la isla. Sin embargo, para efectos de nuestra investigación, nos concentramos en tres textos publicados y traducidos al inglés que se publicaron en la primera década del siglo XXI: en los que se resalta la gastronomía puertorriqueña: Cocina artesanal puertorriqueña (2004), Puerto Rico: la gran cocina del Caribe (2004) y Sabor criollo (2007). Estos se tradujeron respectivamente como Puerto Rican Artisanal Cooking (2009), Puerto Rico: Grand Cuisine of the Caribbean (2004) y Puerto Rico: True Flavors (2007).

El libro Cocina artesanal puertorriqueña fue escrito por Emma Duprey de Sterling entre los años sesenta y setenta, pero no se publicó hasta 2004, según nos consta por una comunicación personal con Aurora Lauzardo, una de las traductoras del texto. Duprey de Sterling se concentra en ofrecer alternativas de comida sana. Si bien la autora no cuenta con preparación profesional en el ámbito culinario, de acuerdo con la nota de las traductoras que aparece en la versión en inglés, Sterling tuvo un negocio de comidas a domicilio y restaurantes $(2009,7)$, por lo que tiene un nivel de especialización intermedio.

Puerto Rico: la gran cocina del Caribe, editado por el crítico gastronómico José Díaz de Villegas, cuenta con recetas de chefs de origen puertorriqueño o residentes en la isla. José Luis Díaz de Villegas, hasta su fallecimiento considerado la autoridad en temas gastronómicos del país, publicaba una columna sobre temas culinarios en el periódico El Nuevo Día y fue autor de varios libros sobre gastronomía. Los chefs cuyas recetas aparecen en este texto estudiaron en prestigiosos institutos culinarios y se han

Ismarie DIÁZ FLORES

La terminología de la gastronomía puertorriqueña: reflexiones en torno a su trasfondo y representación
CLINA

vol. 4-2, December 2018, 181-205

elSSN: 2444-1961

Ediciones Universidad de Salamanca - CC BY-NC-ND 
destacado en la gastronomía local e internacional. El texto presenta recetas complejas y se concentra en la alta cocina.

Sabor criollo fue escrito por el chef Wilo Benet -quien también participó en el texto editado por Díaz De Villegas-. Sin embargo, Sabor criollo presenta un bajo nivel de especialización. Al igual que los textos publicados en los años cincuenta, en estos tres textos está presente el interés por definir una comida típica puertorriqueña, ya sea elevándola a categoría gourmet o acercándola al público lego.

Al acercarnos a estos textos, debemos tomar en cuenta, de una parte, que también pueden caracterizarse como manifestaciones del nacionalismo cultural. De otra parte, debemos considerar el auge del movimiento foodie. De acuerdo con Johnston y Baumann (2014), los foodies se perciben como aficionados a la gastronomía que entienden el comer como un arte, pero que se caracterizan por gravitar en torno a lo que consideran auténtico o exótico. Los investigadores describen a los foodies como personas motivadas tanto por la búsqueda de emociones fuertes como por la demostración de su sofisticación y estatus social.

Johnston y Bauman observan que en el contexto foodie se palpa tanto un interés por establecer vínculos y aprender de otras culturas como la reproducción de desigualdades neocoloniales. Estas tensiones adquieren otros matices entre foodies pertenecientes a clases sociales y trasfondos étnicos y culturales diversos (Oleschuk 2017). La imagen de la cocina puertorriqueña que se presenta en los textos antes mencionados puede ser consumida por foodies puertorriqueños, tanto en la isla como en la diáspora, que desde la perspectiva de la autenticidad buscan conectarse con sus raíces. Asimismo, pueden ser leídos por foodies extranjeros, que perciben la cocina puertorriqueña como extensión del Otro exótico.

En nuestro corpus, solo incluimos recetas de autores puertorriqueños, por lo que no incluimos aquellas de Puerto Rico: la gran cocina del Caribe que no cumplieran tal condición. Dado que queríamos buscar posibles equivalentes en inglés de los términos, solo incorporamos las recetas que contaban con una traducción. Excluimos las recetas de postres y bebidas. El resultado de este proceso de selección es un corpus paralelo con un total de 600 recetas, cuyas características se desglosan en las tablas 1 y 2. Para su estudio, utilizamos la herramienta de análisis de corpus Wordsmith Tools 6.0 .

\begin{tabular}{|l|l|l|}
\hline \multicolumn{3}{|c|}{ Corpus de recetas en español } \\
\hline Libro & Número de recetas & Número de tokens \\
\hline Cocine a gusto & 172 & 16437 \\
\hline Cocina criolla & 176 & 41701 \\
\hline Puerto Rico Sabor criollo & 111 & 35150 \\
\hline
\end{tabular}

Ismarie DIAZZ FLORES

La terminología de la gastronomía puertorriqueña: reflexiones en torno a su trasfondo y representación 


\begin{tabular}{|l|l|l|}
\hline \multicolumn{3}{|c|}{ Corpus de recetas en español } \\
\hline Libro & Número de recetas & Número de tokens \\
\hline Cocina artesanal puertorriqueña & 106 & 23390 \\
\hline Puerto Rico. La gran cocina del Caribe & 35 & 13009 \\
\hline Totales & 600 & 129687 \\
\hline
\end{tabular}

Tabla 1: Composición del corpus de recetas en español

\begin{tabular}{|l|l|l|}
\hline \multicolumn{3}{|c|}{ Corpus de recetas en inglés } \\
\hline Libro & Número de recetas & Número de tokens \\
\hline Puerto Rican Dishes & 172 & 16003 \\
\hline Puerto Rican Cooking & 176 & 32572 \\
\hline Puerto Rico. True Flavors & 111 & 32883 \\
\hline Puerto Rican Artisanal Cooking & 106 & 18944 \\
\hline Puerto Rico: Grand Cuisine of the Caribbean & 35 & 11085 \\
\hline Totales & 600 & 111487 \\
\hline
\end{tabular}

Tabla 2: Composición del corpus de recetas en inglés

\subsection{Selección y análisis de las unidades terminológicas}

De acuerdo con la TbM, la terminología de un dominio puede identificarse y definirse a partir del marco del proceso clave de este dominio. El evento se plantea como un marco que contiene las categorías conceptuales principales del dominio y las relaciones que guardan entre sí (Faber et al. 2006: 191). A fin de facilitar la identificación de unidades terminológicas, delimitamos el marco del proceso culinario a partir de múltiples fuentes documentales que, posteriormente, contrastamos con los datos de nuestro corpus. Entre estas fuentes, revisamos lenguajes de marcado de recetas como Recipe Markup Language (RecipeML) (Saiya 2002); Recipe Exchange Markup Language (REML), diseñado por Gary Gocek (SourceForge 2013); y hRecipe (MicroFormats 2008), Rich Snippets de Google y el asociado a la BBC Food Ontology. Asimismo, examinamos diversas ontologías como Ontochef (Paulo Pardal y Mamede 2011, Ribeiro et al. 2006 y Batista et al. 2006) y las presentadas en Nanba et al. (2014) y Sam et al. (2014). La comparación entre las categorías utilizadas en estos lenguajes de marcado y ontologías nos permitieron identificar una serie de categorías 
conceptuales básicas presentes en las recetas, las cuales utilizamos en la creación del marco del evento culinario. Otros recursos útiles en este proceso lo fueron libros sobre procesos de cocina (Gil Martínez 2010, Pérez Oreja et al. 2010).

También verificamos los recursos del proyecto Taaable (Dufour-Lussier et al. 2012, Champin et al. 2008) y los lenguaje de representación semántica Minimal Instruction Language for the Kitchen (MILK), (Tasse y Smith 2008), Simplified Ingredient Merging Map in Recipes (SIMMR) (Jermsurawong y Habash 2015) y el descrito en Malmaud et al. (2014). Asimismo, consideramos las categorías en la ontología JaDaCook (Herrera et al. 2009). También adaptamos las categorías de modificadores verbales identificadas por Karlin en el desarrollo del proyecto SEAFACT (1988: 14-32). A continuación presentamos dos tablas comparativas en las que se ilustran las categorías presentes en los lenguajes de marcado y en las ontologías.

\begin{tabular}{|c|c|c|c|c|}
\hline \multirow{2}{*}{$\begin{array}{l}\text { Tipo de } \\
\text { categoría }\end{array}$} & \multicolumn{4}{|c|}{ Lenguaje de marcado } \\
\hline & REML & hRecipe & $\begin{array}{l}\text { Google Rich } \\
\text { Snippets }\end{array}$ & $\begin{array}{l}\text { BBC Food } \\
\text { Ontology }\end{array}$ \\
\hline Título & recipemIElement & $F n$ & name & Recipe \\
\hline Ingredientes & ingredientsElement & ingredient & ingredients & $\begin{array}{l}\text { Ingredient } \\
\text { IngredientList } \\
\text { Food } \\
\text { ShoppingCategory }\end{array}$ \\
\hline Porciones & yieldElement & Yield & recipeYield & \\
\hline Procedimiento & directiondfsElement & instructions & recipelnstructions & $\begin{array}{l}\text { Stage } \\
\text { StageSequence } \\
\text { Step } \\
\text { Technique } \\
\text { Method }\end{array}$ \\
\hline $\begin{array}{l}\text { Momento de la } \\
\text { comida en que } \\
\text { se sirve }\end{array}$ & - & - & recipeCategory & Course \\
\hline $\begin{array}{l}\text { Tiempo de } \\
\text { preparación }\end{array}$ & preptimeElement & - & $\begin{array}{l}\text { prepTime } \\
\text { cookTime } \\
\text { totalTime }\end{array}$ & - \\
\hline Equipo & $\begin{array}{l}\text { equipmentElement } \\
\text { toolElement }\end{array}$ & - & - & - \\
\hline $\begin{array}{l}\text { Información } \\
\text { relacionada } \\
\text { con la } \\
\text { nutrición }\end{array}$ & - & - & nutrition & Diet \\
\hline
\end{tabular}

Ismarie DIAZZ FLORES La terminología de la gastronomía puertorriqueña: reflexiones en torno a su trasfondo y representación 


\begin{tabular}{|l|c|l|l|l|}
\hline $\begin{array}{l}\text { Tipo de } \\
\text { categoría }\end{array}$ & Lenguaje de marcado & - & $\begin{array}{l}\text { Menu } \\
\text { Occasion } \\
\text { Season }\end{array}$ \\
\hline $\begin{array}{l}\text { Tipo de receta } \\
\text { por período de } \\
\text { consumo }\end{array}$ & - & - & - & Collection \\
\hline $\begin{array}{l}\text { Tipo de } \\
\text { receta por } \\
\text { ingrediente }\end{array}$ & - & - & - & Cuisine \\
\hline $\begin{array}{l}\text { Tipo de receta } \\
\text { por región }\end{array}$ & - & - & - & \\
\hline $\begin{array}{l}\text { Datos de } \\
\text { identificación }\end{array}$ & - & - & $\begin{array}{l}\text { description } \\
\text { datePublished } \\
\text { author }\end{array}$ & \\
\hline Evaluación & - & - & review & - \\
\hline
\end{tabular}

Tabla 3: Comparación de lenguajes de marcado

\begin{tabular}{|l|l|l|l|l|l|}
\hline $\begin{array}{l}\text { Tipo de } \\
\text { categoría o } \\
\text { relación }\end{array}$ & \multicolumn{2}{|l|}{ Ontología } \\
\hline & Ontochef & Sam et al. & Taaable & Nanba et al. & JaDaCook \\
\hline $\begin{array}{l}\text { Título / } \\
\text { Plato }\end{array}$ & Receita & hasName & $\begin{array}{l}\text { Recipe } \\
\text { Dish }\end{array}$ & - & - \\
\hline $\begin{array}{l}\text { Relación } \\
\text { ingrediente- } \\
\text { plato }\end{array}$ & - & produces & & - & $\begin{array}{l}\text { Contains } \\
\text { Title } \\
\text { Contains } \\
\text { Alimento }\end{array}$ \\
$\begin{array}{l}\text { Alimento } \\
\text { Alimento } \\
\text { Parte de } \\
\text { alimento } n \text { to } \\
\text { sólido } \\
\text { Estado de } \\
\text { alimento } \\
\text { Alimento } \\
\text { com estado }\end{array}$ & & Food & & \\
\hline
\end{tabular}




\begin{tabular}{|c|c|c|c|c|c|}
\hline $\begin{array}{l}\text { Tipo de } \\
\text { categoría o } \\
\text { relación }\end{array}$ & Ontología & & & & \\
\hline Ingredientes & $\begin{array}{l}\text { Ingredientes } \\
\text { necesários } \\
\text { Ingredientes } \\
\text { produzidos } \\
\text { Preparação }\end{array}$ & hasIngredient & $\begin{array}{l}\text { IngredientFood } \\
\text { Ingredient }\end{array}$ & $\begin{array}{l}\text { Ingredient- } \\
\text { seafood } \\
\text { Ingredient- } \\
\text { meat } \\
\text { Ingredient- } \\
\text { vegetable } \\
\text { Ingredient- } \\
\text { other } \\
\text { Condiment }\end{array}$ & $\begin{array}{l}\text { Animal } \\
\text { Origin } \\
\text { Plant Origin } \\
\text { Sweteners, } \\
\text { Drinks } \\
\text { Basic }\end{array}$ \\
\hline $\begin{array}{l}\text { Atributos de } \\
\text { ingrediente }\end{array}$ & - & $\begin{array}{l}\text { consistsOf } \\
\text { classifiedAs }\end{array}$ & - & - & - \\
\hline $\begin{array}{l}\text { Unidades de } \\
\text { medida }\end{array}$ & $\begin{array}{l}\text { Medição } \\
\text { Unidades e } \\
\text { Medidas } \\
\text { Equivalência }\end{array}$ & - & $\begin{array}{l}\text { Quantity } \\
\text { Unit } \\
\text { MassUnit } \\
\text { TemperatureUnit } \\
\text { TimeUnit } \\
\text { VolumeUnit }\end{array}$ & - & - \\
\hline Utensilios & Utensilios & - & Utensil & Kitchen tool & - \\
\hline $\begin{array}{l}\text { Relación } \\
\text { utensilio- } \\
\text { receta }\end{array}$ & - & Requires & - & - & - \\
\hline Fases & $\begin{array}{l}\text { Preparação } \\
\text { Confecção } \\
\text { Apresentação }\end{array}$ & - & - & - & - \\
\hline Acciones & $\begin{array}{l}\text { Acção } \\
\text { Acção } \\
\text { ordenada } \\
\text { Acção } \\
\text { simples } \\
\text { Acção } \\
\text { composta }\end{array}$ & & $\begin{array}{l}\text { RecipeAction } \\
\text { RecipeSimple } \\
\text { Action } \\
\text { RecipeComplex } \\
\text { Action }\end{array}$ & Movement & - \\
\hline $\begin{array}{l}\text { Relación } \\
\text { acción- } \\
\text { descripción }\end{array}$ & - & $\begin{array}{l}\text { hasProcess } \\
\text { Description } \\
\text { hasTextual D } \\
\text { escription }\end{array}$ & - & - & - \\
\hline
\end{tabular}

Ismarie DÍAZ FLORES La terminología de la gastronomía puertorriqueña: reflexiones en torno a su trasfondo y representación 


\begin{tabular}{|l|l|l|l|l|l|}
\hline $\begin{array}{l}\text { Tipo de } \\
\text { categoría o } \\
\text { relación }\end{array}$ & \multicolumn{5}{|l|}{ Ontología } \\
\hline $\begin{array}{l}\text { Modificadores } \\
\text { o condiciones } \\
\text { de acciones }\end{array}$ & $\begin{array}{l}\text { duração } \\
\text { (Tarefa) } \\
\text { quantidade } \\
\text { (Ingrediente) } \\
\text { necessários } \\
\text { produzidos } \\
\text { (Ingrediente) }\end{array}$ & $\begin{array}{l}\text { preparationTime } \\
\text { cookingTime }\end{array}$ & $\begin{array}{l}\text { ActionParameter } \\
\text { ActionQualifier } \\
\text { StopCondition } \\
\text { Until } \\
\text { During } \\
\text { Duration }\end{array}$ & - & \\
\hline $\begin{array}{l}\text { Datos de } \\
\text { identificación }\end{array}$ & - & $\begin{array}{l}\text { has/nformation } \\
\text { Object } \\
\text { hasURL } \\
\text { hasKeyword } \\
\text { hasAuthor } \\
\text { hasRecommender } \\
\text { hasDifficultyLevel }\end{array}$ & - & - \\
\hline Evaluación & - & hasRating & - & & \\
\hline
\end{tabular}

Tabla 4: Comparación de ontologías

Por medio de estos recursos, identificamos una serie de categorías básicas que formaban parte del marco del evento culinario: PROCESOS, INGREDIENTES, UTENSILIOS, UNIDADES DE MEDIDA y PLATOS. Posteriormente, contrastamos y perfeccionamos la delimitación de las categorías a partir de los datos obtenidos por medio del corpus. La selección de las unidades se llevó a cabo en varias etapas, tomando en consideración la frecuencia de uso, la presencia en el contexto del marco del evento culinario y la relevancia de los términos a la luz del contexto cultural.

\subsection{Diseño de la base de datos terminológica}

La última etapa de este proceso consistía en diseñar una base de datos que nos permitiera representar los diferentes niveles de contexto. La base de datos terminológica se preparó por medio del programa MultiTerm. Cada término se clasificó dentro de una de las siguientes categorías conceptuales: INGREDIENTE, UTENSILIO, UNIDAD DE MEDIDA, PROCESO, PLATO Y PROPIEDADES y ESTADOS. La selección final de categorías conceptuales a que incluir en la base de datos responde, por un lado, a las categorías prevalecientes en diversos libros de referencia, bases de datos, ontologías y lenguajes de marcado asociados con alimentación y, por otro lado, a los hallazgos del corpus.

Las entradas de la base de datos cuentan con una definición y campos con información gramatical, desglose de las relaciones conceptuales pertinentes para cada 
categoría y ejemplos de uso. En la explicitación de las relaciones conceptuales, se incluyen enlaces a los términos con los cuales se establecen las relaciones conceptuales. Debemos mencionar que otro recurso útil en la delimitación de subcategorías dentro de la categoría de PLATO fueron los índices de los libros de recetas, en los que se indicaba a qué tipo de preparación pertenecía cada receta.

\section{DISCUSIÓN}

\subsection{Las viandas: uno de los componentes clave de la dieta puertorriqueña}

Si bien como parte de nuestra investigación identificamos y analizamos términos de diversas categorías conceptuales, como ingredientes, procesos y utensilios, entre otros, en los siguientes apartados nos concentraremos en la aplicación de nuestro aparato teórico en un subconjunto de ingredientes: las viandas. Era Pérez (2003) propone la siguiente definición de vianda como parte de un estudio de esta unidad léxica en la variante cubana del español:

vianda f. Cuba y P. Ric. (Culinaria y Agricultura) Vegetales con los que es posible hacer papillas o purés y pueden ser tubérculos, raíces o frutos que se consumen cocidos, fritos o en sopas. / El ajiaco cubano incluye viandas como la calabaza, la yuca, la papa y muchas otras (Era Pérez 2003, 74).

Como indica la investigadora, este es un término que reúne un conjunto de alimentos, semejantes en función de los tipos de preparaciones en los que aparecen. Elegimos estos términos para ilustrar la aplicación de nuestro método ya que el análisis a la luz de los múltiples niveles de contexto nos permite entender cómo terminan por incluirse bajo una misma categoría alimentos que no pertenecen a la misma clasificación desde una perspectiva botánica y que pueden prepararse por medio de cualquiera de las principales técnicas de cocción. De hecho, tal y como señala Roberts, podemos acercarnos a esta categoría de ingredientes desde una perspectiva social:

Viandas es un término empleado para cubrir un grupo de tubérculos o vegetales amiláceos tales como yuca, yautía, malanga, ñame, así como también las frutas amiláceas y panapén. Éstas son ampliamente usadas en Puerto Rico, y en hogares de bajos ingresos, constituyen la mayoría de la dieta, sean solas o acompañadas con arroz y habichuelas. [...] (Roberts 1949, en Ortiz Cuadra 2006, 171).

Ismarie DÍAZ FLORES

La terminología de la gastronomía puertorriqueña: reflexiones en torno a su trasfondo y representación 
Ortiz Cuadra (2006) explica que las viandas se volvieron un elemento clave en la estructuración de los platos en la gastronomía puertorriqueña por su resistencia, capacidad de reproducción y accesibilidad. Según destaca el investigador, esta era una característica clave en el Puerto Rico preindustrial, ya que entonces imperaba el monocultivo, así como en periodos posteriores, en los que se incrementó la dependencia en la importación. En este punto, comenzamos a ver cómo se conjugan varios elementos en la configuración de la categoría, como la disponibilidad de estos alimentos y el uso que en el contexto histórico social se hace de estos. A estos aspectos se suma la forma en que se representan los miembros de esta categoría en la producción textual.

\subsection{Las viandas en el corpus}

Al analizar las concordancias relacionadas con las viandas en el corpus, encontramos otros datos valiosos respecto a las relaciones que establecen entre sí como categoría y con ciertos procedimientos, utensilios y productos. El término vianda aparece en nuestro corpus 47 veces. En cuatro ocasiones, como parte del título de recetas: "Guiso de viandas", "Sopa de viandas», "Viandas majadas» y "Viandas hervidas». También encontramos incisos en los que se especifica qué productos pertenecen a la categoría: «viandas hervidas: plátanos, batatas, papas, etc.», «Viandas (yautías, batatas, guineos verdes, plátanos, etc.» o en listas que incluían numerosos productos. Asimismo, encontramos la indicación de que se cocinen «hasta que estén tiernas». Finalmente, en quince ocasiones, el término aparece como objeto de procesos: añadir (3 veces), escurrir (4 veces), rallar (2 veces), colocar (2), hincar (1 vez), majar (1 vez), mondar (1 vez), preparar (1 vez). El término aparece modificado por majadas (4 veces), hervidas (3 veces) y cremosas (1). Al revisar las colocaciones para estos procesos, encontramos que existía afinidad entre varios de estos (p. ej., rallar, mondar) y los miembros de la categoría vianda. Por ejemplo, encontramos oraciones como: «Monde el ñame y hierva en agua con sal hasta que esté blando [...]» (Cabanillas et al. 1983 [1950], 113) o «Lave y monde las yautías» (Aboy de Valldejuli [1954 (2011)], 223).

Este análisis arroja luz respecto las características de este tipo de ingrediente y las relaciones que puede establecer con otros conceptos, lo que nos ayuda a comprender fenómenos como cambios en la extensión de los términos o la incorporación de términos nuevos. Por ejemplo, mofongo denomina un plato hecho a base de plátano verde frito majado, mezclado con algún tipo de grasa y un elemento crujiente (usualmente, chicharrón de cerdo). Este plato aparece tanto en los libros de recetas de los años cincuenta como en los más recientes, preparado con los mismos ingredientes y procesos básicos. Sin embargo, en Sabor criollo, encontramos las recetas «Mofongo

Ismarie DIAZ FLORES

La terminología de la gastronomía puertorriqueña: reflexiones en torno a su trasfondo y representación
CLINA

vol. 4-2, December 2018, 181-205

elSSN: 2444-1961

Ediciones Universidad de Salamanca - CC BY-NC-ND 
de amarillos»² (Benet 2009, 55) y "Mofongo de yuca» (Ibíd.: 59). Estas y otras versiones no registradas en estos textos -como el trifongo, plato en el que se incorporan plátanos verdes, maduros y yuca- también se encuentran disponibles en restaurantes y se mencionan en páginas web ${ }^{3}$. A partir de nuestra investigación, entendemos que estas nuevas versiones se insertan de forma coherente en el sistema conceptual, lo que, a su vez se refleja en la creación de nuevos términos.

El plátano con el que se prepara la versión original del mofongo es una vianda, que comparte propiedades intrínsecas (p. ej. textura, contenido de almidones) y extrínsecas (p. ej. platos en los que se utilizan, procesos asociados) con los demás alimentos que forman parte de esta categoría. Las nuevas versiones de este plato sustituyen el plátano por otra vianda o incorporan varias. En cuanto a los términos, observamos el uso de procedimientos comunes de formación de términos, tanto en el contexto del español como en el ámbito específico de la cocina, tal y como se describen en investigaciones como Viudas Camarasa (1982) o Navarro (2008). Por otro lado, en la versión en inglés del texto de Benet, observamos que los términos utilizados para denominar los diversos tipos de mofongo son respectivamente green plantain mofongo, ripe plantain mofongo y cassava mofongo. Si bien se conserva el préstamo mofongo, se indica la vianda utilizada en cada caso.

\subsection{Las viandas en el contexto de la lengua española}

Si bien nuestra investigación se concentra en el estudio de la terminología utilizada en el contexto de los libros de recetas de Puerto Rico, nos pareció pertinente situar los términos relacionados a esta categoría en el contexto más amplio de la lengua española. Con este propósito, nos referimos al Diccionario crítico etimológico de la lengua castellana (DCECH) (Corominas 1954) y al Corpus del Nuevo Diccionario Histórico del Español (CDH), a fin de obtener datos sobre las primeras apariciones documentadas de estos términos en el ámbito del español.

Tras revisar estas fuentes, encontramos que la mayoría aparece por primera vez en textos de los cronistas españoles y en textos de tema histórico. En algunos casos, como en los de apio, pana y papa, e incluso, el propio término vianda, resultó difícil dar con una primera aparición del término a causa de la existencia de homónimos. En el caso de apio, debemos hacer la salvedad de que, aunque existe una entrada en el DCECH, esta no se refiere a la misma planta, si bien ambas son de la familia de las apiáceas. Debemos enfatizar que este es solo un primer acercamiento para ubicar el

2. En el contexto de la gastronomía puertorriqueña, el término amarillo es sinónimo de plátano maduro.

3. Véase, por ejemplo https://www.sal.pr/recipe/receta-del-dia-trifongo.

Ismarie DIAZZ FLORES

La terminología de la gastronomía puertorriqueña: reflexiones en torno a su trasfondo y representación 
uso de estos términos en otros países de habla hispana, aunque en un futuro, nos interesa explorar el tema por medio de fuentes más específicas de Puerto Rico y el Caribe.

\begin{tabular}{|c|c|c|}
\hline Término & DCECH & $\mathrm{CDH}$ \\
\hline apio & 1. a doc.: 1423 , Villena & $\begin{array}{l}1573 \text { Aguado, Fray Pedro de, Historia } \\
\text { de Santa Marta y Nuevo Reino de } \\
\text { Granada [Colombia] [Jerónimo Bécker, } \\
\text { Madrid, Establecimiento Tipográfico } \\
\text { de Jaime Ratés, 1916-1917] Historia }\end{array}$ \\
\hline batata & $\begin{array}{l}\text { 1.a doc. } 1519 \text { (V. Hz. Ureña, p. 66); } \\
\text { el italiano Pedro Mártir de Anghiera } \\
\text { ya la menciona en latín en } 1516 .\end{array}$ & $\begin{array}{l}1544 \text { Anónimo. Segunda relación } \\
\text { anónima de la jornada que hizo Nuño } \\
\text { de Guzmán }\end{array}$ \\
\hline chayote & $\begin{array}{l}\text { 1.a doc.: } 1767 \text { Esteban de Terreros } \\
\text { y Pando, Diccionario Castellano } \\
\text { de las Voces de Ciencias y Artes y } \\
\text { sus Correspondientes en las tres } \\
\text { lenguas francesa, latina e italiana }\end{array}$ & $\begin{array}{l}1560 \text { Cervantes De Salazar, Francisco, } \\
\text { Crónica de la Nueva España [España] } \\
\text { [Manuel Magallón, Madrid, Atlas, } \\
\text { 1971] Historia }\end{array}$ \\
\hline malanga & No documentado. & $\begin{array}{l}\text { 1840-a1862 El Cucalambé (Juan } \\
\text { Cristóbal Nápoles Fajardo), Poesías } \\
\text { completas [Cuba] [Jesús Orta Ruiz, La } \\
\text { Habana, Editorial de Arte y Literatura, } \\
\text { 1974] Verso }\end{array}$ \\
\hline ñame & 1.a doc.: 1492, Colón (niame). & $\begin{array}{l}\text { 1535-1557 Fernández De Oviedo, } \\
\text { Gonzalo, Historia general y natural } \\
\text { de las Indias [España] [Juan Pérez de } \\
\text { Tudela Bueso, Madrid, Atlas, 1992] } \\
\text { Biología }\end{array}$ \\
\hline papa & $\begin{array}{l}\text { 1.a doc.: h. } 1540 \text {, con referencia al } \\
\text { Valle del Cauca (Sur de Colombia). }\end{array}$ & $\begin{array}{l}1553 \text { Cieza De León, Pedro, Crónica } \\
\text { del Perú [Perú] [Carmelo Sáenz de } \\
\text { Santa María, Madrid, CSIC, 1984] } \\
\text { Historia }\end{array}$ \\
\hline pana & No documentado. & $\begin{array}{l}1986 \text { García Ramis, Magali, Felices } \\
\text { días tío Sergio [Puerto Rico] [ Georgia, } \\
\text { Antillana, 1995] Novela }\end{array}$ \\
\hline $\begin{array}{l}\text { plátano } \\
\text { verde }\end{array}$ & No documentado. & $\begin{array}{l}1748 \text { Juan, Jorge/Ulloa, Antonio De, } \\
\text { Discurso y reflexiones políticas sobre } \\
\text { el estado presente de la marina en los } \\
\text { reinos del Perú [Perú] [Luis J. Ramos } \\
\text { Gómez, Madrid, CSIC, 1985] }\end{array}$ \\
\hline
\end{tabular}

Ismarie DIÁZ FLORES La terminología de la gastronomía puertorriqueña: reflexiones en torno a su trasfondo y representación 


\begin{tabular}{|l|l|l|}
\hline Término & DCECH & CDH \\
\hline yautía & No documentado. & $\begin{array}{l}\text { 1535-1557 Fernández De Oviedo, } \\
\text { Gonzalo, Historia general y natural } \\
\text { de las Indias [España] [Juan Pérez de } \\
\text { Tudela Bueso, Madrid, Atlas, 1992] } \\
\text { Biología }\end{array}$ \\
\hline yuca & 1.a doc.: h 1495, Fr. Ramón Pané(r). & $\begin{array}{l}\text { 1495 ANÓNIMO, «Carta de Colón } \\
\text { los Reyes»(Textos y documentos } \\
\text { completos de Cristóbal Colón) } \\
\text { [España] [, , ] Testimonios varios }\end{array}$ \\
\hline vianda & No documentado. & $\begin{array}{l}\text { 1938 SERPA, ENRIQUE, } \\
\text { Contrabando. Novela [Cuba] [Néstor } \\
\text { A. Moreno, Miami, Ediciones } \\
\text { Universal, 1973] Novela }\end{array}$ \\
\hline
\end{tabular}

Primera datación de los términos en el DCECH y el CDH

\subsection{Representación de la información en la base de datos}

En la entrada del término vianda en la base de datos incluimos una definición que incorpora los datos obtenidos por medio del corpus. Incluimos la extensión del término e indicamos las principales técnicas asociadas con la preparación de este tipo de ingrediente. Asimismo, señalamos las relaciones conceptuales pertinentes para la categoría: HIPÓNIMOS, INGREDIENTE-DE y PROCESOS ASOCIADOS. Finalmente, incluimos ejemplos de uso que aportan más información respecto a los procesos y cualidades asociados a esta categoría. En el caso de la relación INGREDIENTE-DE, por ejemplo, incluimos aquellos platos que aparecían en más de un texto, en los que se utilizaban viandas variadas: masa, pastelón y serenata de bacalao. En el caso de viandas específicas (p. ej. yuca, plátano, ñame, etc.), también incluimos esta relación, pero indicamos los platos hechos a partir de esas viandas en particular.

Ismarie DIAZ FLORES

La terminología de la gastronomía puertorriqueña: reflexiones en torno a su trasfondo y representación 


\section{Spanish (Puerto Rico)}

vianda

Tipo: forma completa

Categoría gramatical: $\mathrm{n}$.

Género gramatical: f.

Número: sing.

Definición: Género de raíces comestibles, tubérculos y

frutas que incluye el apio, la batata, la

calabaza, el ñame, los plátanos, los guineos

verdes, la papa, la yautía y la yuca, que

suelen consumirse hervidas o majadas y que

se utilizan ralladas para preparar masas.

Relaciones

conceptuales:

hipónimos: apio, batata, chayote,

guineo verde, malanga, ñame, pana,

papa, plátano, yautía, yuca

procesos asociados: mondar, hervir,

rallar, majar

ingrediente de: masa, pastelón,

serenata de bacalao

Ejemplo de Sirva con viandas hervidas: plátanos,

uso:

batata, papas, etc.

\section{Fuente: CG_ES_129}

Ejemplo de Agregue la mantequilla y bátala hasta que uso: $\quad$ se derrita en el majado de viandas.

\section{Fuente: SC_ES_33}

Ejemplo de Coloque un poco de vianda majada en el uso: fondo de un plato $[\ldots]$.

Figura 1: Entrada de la base de datos en español para vianda

La entrada también incluye la información correspondiente en inglés, a partir de los hallazgos del corpus. En este caso, por no haber un equivalente exacto, sugerimos el uso del préstamo, procedimiento utilizado en algunas de las recetas del corpus. No obstante, la inclusión de los hipónimos y, en el caso de los términos que denominan viandas específicas, la inclusión de otros posibles hiperónimos, brinda al traductor la posibilidad de utilizar otro término en función de las necesidades de la traducción. Por ejemplo, en el caso de la yuca, desde la perspectiva botánica, se clasifica como una raíz tuberosa, hiperónimo que incluimos en la entrada en español junto con vianda. Asimismo, incluimos los hipónimos para yuca tanto en la categoría de vianda como en la de raíz tuberosa. Del mismo modo, en la entrada en inglés, indicamos los hiperónimos tuberous root y root vegetable. Como complemento a estas informaciones, incluimos también fotos de cada una de las viandas individuales. 


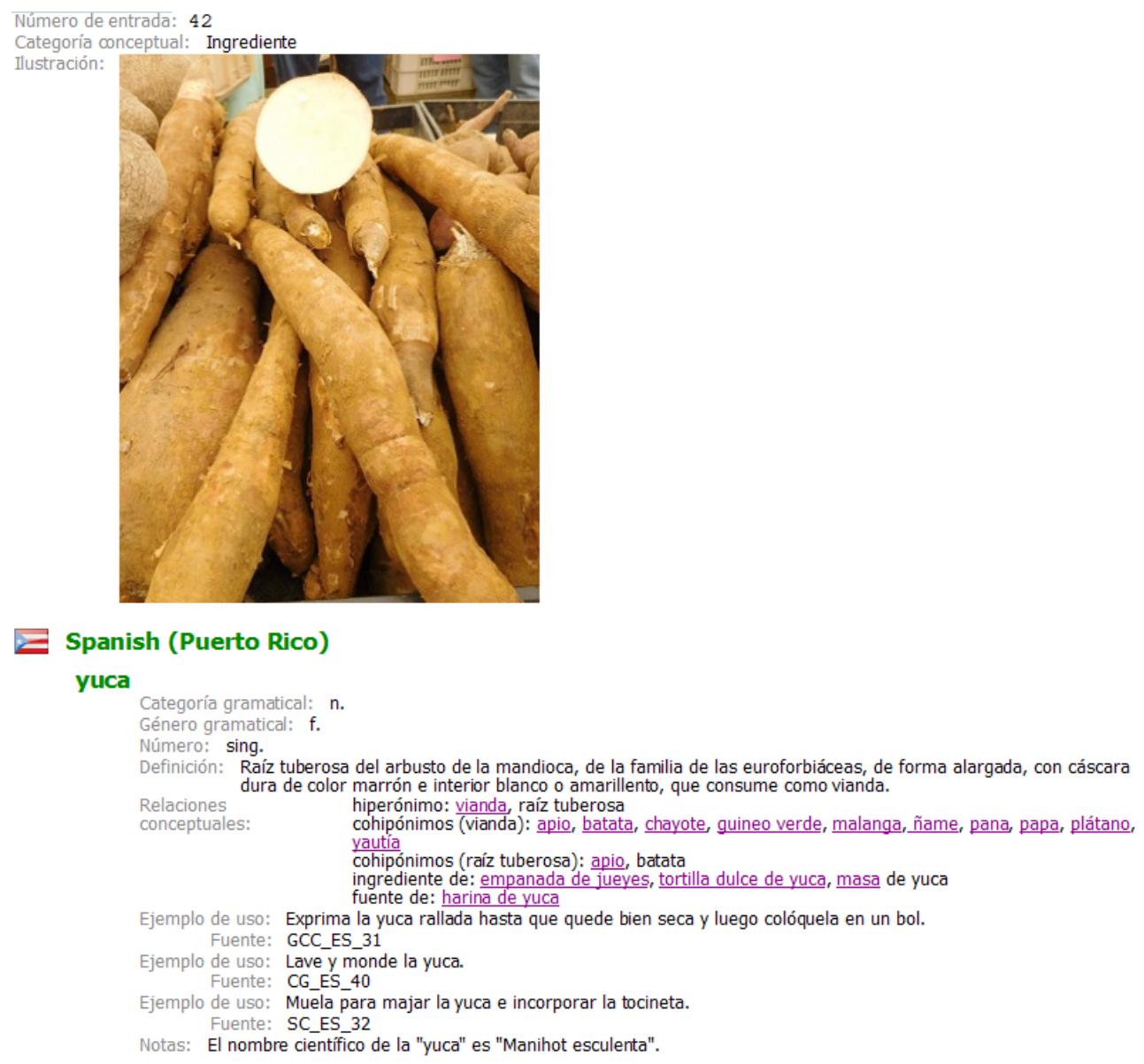

Figura 2: Entrada de la base de datos en español para yuca

\section{CONCLUSIÓN}

El conocimiento de la terminología culinaria resulta de vital importancia en el contexto de la comunicación intercultural. Como mencionáramos antes, actualmente se producen una variedad de textos, como videos, blogs y programas televisivos, en los que se transmiten conocimientos culinarios de diversas culturas. Asimismo, nos consta que como resultado de los procesos de emigración y el establecimiento de restaurantes puertorriqueños en otras latitudes, existen múltiples contextos en los que los platos de esta cocina se consumen «en traducción».

Ismarie DÍAZ FLORES

La terminología de la gastronomía puertorriqueña: reflexiones en torno a su trasfondo y representación 
Ante este panorama de intercambios culturales constantes, no podemos acercarnos a los términos relacionados con la cocina -o mejor, cocinas- de manera aislada, sino como parte de un sistema. Dicho sistema y la terminología asociada a este son el resultado de una larga historia de choques e intercambios culturales que han dado lugar a diferentes formas de categorizar y conceptualizar. Por medio de nuestra investigación, hemos intentado dar cuenta de cómo los distintos niveles de contexto en los que se inserta la terminología de la gastronomía puertorriqueña, como lo son el entorno, el contexto histórico, la producción textual y las particularidades del dominio culinario, se han plasmado en la configuración de categorías y el uso de la terminología. A fin de ilustrar lo anterior, nos hemos centrado en una categoría utilizada en la gastronomía puertorriqueña -así como en otros países caribeños-, aquella de las viandas.

A partir de lo antes expuesto, concluimos que siempre que estudiamos terminología culinaria, lo hacemos en el contexto de una cultura. El estudio minucioso del sistema conceptual culinario a partir de las herramientas teóricas que nos ofrece la Terminología y el desarrollo de recursos terminológicos que representen los diversos niveles de significación asociados a los términos ofrece al traductor una perspectiva amplia desde la cual acercarse a los textos ligados a la gastronomía.

\section{BIBLIOGRAFÍA}

APPADURAl, Arjun. 1988. «How to Make a National Cuisine: Cookbooks in Contemporary India». Comparative Studies in Society and History 30 (1): 3-24.

AYORA DÍAZ, Steffan Igor. 2017. «Translocalidad, globalización y regionalismo: cómo entender la gastronomía regional yucateca». Anales de Antropología 51 (2): 96-105, doi:10.1016/j. antro.2017.03.004.

BAK-GELLER CORONA, Sarah. 2013. "Narrativas deleitosas de la nación. Los primeros libros de cocina en México (1830-1890)». Desacatos 43 (septiembre-diciembre): 31-44.

BARRADAS, Efraín. 2010. «El cocinero puertorriqueño, El manual del cocinero cubano y la formación del nacionalismo en el Caribe». Foro Hispánico 39: 267-279.

Benet, Wilo. 2009. Puerto Rico True Flavors. 3. ${ }^{a}$ ed. Trad. Rodrigo López Chávez. Baltimore, Maryland, Estados Unidos: Read Street Publishing Inc.

BENET, Wilo. 2009. Puerto Rico Sabor criollo. 3. ${ }^{2}$ ed. Baltimore, Maryland, Estados Unidos: Read Street Publishing Inc.

CABANILLAS DE RODRíGuEZ, Berta. 1973. El puertorriqueño y su alimentación a través de su historia (siglos XVI al XIX). San Juan: Instituto de Cultura Puertorriqueña.

CABANILLAS DE RODRíGUEZ, Berta. 1983. El folklore en la alimentación puertorriqueña. Río Piedras: Editorial de la Universidad de Puerto Rico.

CABANILLAS, Berta, Carmen GINORIO y Carmen QUIRÓS. 1983. Cocine a gusto. 15. a ed. San Juan: La Editorial de la Universidad de Puerto Rico.

CABANILLAS, Berta y Carmen GINORIO. 2011. Puerto Rican Dishes. 5. ${ }^{2}$ ed. San Juan: La Editorial de la Universidad de Puerto Rico.

Ismarie DIÁZ FLORES

La terminología de la gastronomía puertorriqueña: reflexiones en torno a su trasfondo y representación
CLINA

vol. 4-2, December 2018, 181-205

elSSN: 2444-1961

Ediciones Universidad de Salamanca - CC BY-NC-ND 
CABRÉ, María Teresa. 2005. La terminología: Representación y comunicación: Elementos para una teoría de base comunicativa y otros artículos. Barcelona: Institut Universitari de Lingüística aplicada, Universitat Pompeu Fabra. Edición Kindle.

CHAMPIN, Pierre-Antoine et al. 2008. "Construction manuelle de la partie haute d'une ontologie modulaire destinée à une annotation de cas textuels - étude de cas pour une application culinaire dans le cadre du projet Taaable». 16ème atelier de Raisonnement à Partir de Cas.

COROMINAS, Joan, 1954. Diccionario crítico etimológico de la lengua castellana. Madrid: Gredos.

DÍAZ FLORES, Ismarie. 2017. La terminología de la gastronomía puertorriqueña y su traducción al inglés. Tesis. Universidad de Salamanca.

DíAZ DE VILLEGAS, José Luis. 2004. Puerto Rico: La gran cocina del Caribe. San Juan: La Editorial de la Universidad de Puerto Rico.

DíAZ DE VILLEGAS, José Luis. 2009. Puerto Rico: Grand Cuisine of the Caribbean. Trad. Madeleine Colón San Juan: La Editorial de la Universidad de Puerto Rico.

DIKI-KIDIRI, Marcel. ed. 2008. Le vocabulaire scientifique dans les langues africaines: pour une approche culturelle de la terminologie. Paris: Karthala.

DuANY, Jorge. 2002. The Puerto Rican Nation on the Move: Identities on the Island \& in the United States. Chapel Hill: University of North Carolina Press.

DUFOUR-LUSSIER, Valmi et al. 2012. «Semi-automatic annotation process for procedural texts: An application on cooking recipes». CoRR abs/1209.5663: n. pag. Consultado el 11 de febrero de 2018. https://arxiv.org/pdf/1209.5663.pdf.

DUPREY DE STERLING, Emma. 2004. Cocina artesanal puertorriqueña. San Juan: Editorial de la Universidad de Puerto Rico.

Duprey DE SteRling, Emma. 2009. Puerto Rican Artisanal Cookery. Trad. Susan Homar y Aurora Lauzardo. La Editorial Universidad de Puerto Rico. San Juan: La Editorial Universidad de Puerto Rico.

ERA PÉREZ, Norma. 2003. «Búsqueda de una definición de la palabra «vianda» en la variante cubana del español». Cultura, economía y desarrollo en Lorca en el alba del siglo XXI: actas del XXXVII Congreso Internacional de la Asociación Europea de Profesores de Español: Lorca, 21-26 de julio de 2002, ed. José Luis Molina Martínez. Editum, 69-75.

FABER, Pamela. 2012. A Cognitive Linguistics View of Terminology and Specialized Language. Berlin; Boston: De Gruyter Mouton.

FABER, Pamela, y Pilar León Araúz. 2014. «Specialized Knowledge Dynamics: From Cognition to Culture-Bound Terminology». En Dynamics and terminology: an interdisciplinary perspective on monolingual and multilingual culture-bound communication, ed. por Rita Temmerman y Marc Van Campenhoudt. Ámsterdam; Filadelfia: John Benjamins Publishing Company (Terminology and Lexicography Researcha and Practice, 16), 135-158.

FABER, Pamela, y M. Carmen ÁFRICA VIDAL CLARAMONTE. 2017. «Food Terminology as a System of Cultural Communication». Terminology, 23 (1): 155-79.

FABER, Pamela et al. 2006. «Process-oriented terminology management in the domain of Coastal Engineering». Terminology 12 (2): 189-213. IngentaConnect.

FERNÁNDEZ-ARMESTO, Felipe. 2004. Historia de la comida: alimentos, cocina y civilización. Trad. Victoria Ordóñez. Barcelona: Tusquets.

FISCHLER, Claude. 1995. El (h)omnívoro: el gusto, la cocina y el cuerpo. Trad. Mario F. Merlino. Barcelona: Anagrama.

Ismarie DIAZ FLORES

La terminología de la gastronomía puertorriqueña: reflexiones en torno a su trasfondo y representación
CLINA

vol. 4-2, December 2018, 181-205

elSSN: 2444-1961

Ediciones Universidad de Salamanca - CC BY-NC-ND 
FLOYD, Janet. 2010. «Simple, Honest Food: Elizabeth David and the Construction of Nation in Cookery Writing». The Recipe Reader: Narratives, Contexts, Traditions, ed. por Janet Floyd y Laurel Forster. Lincoln: University of Nebraska Press, 72-87.

GARZONE, Giuliana. 2017. «Food, Culture, Language and Translation». Journal of Multicultural Discourses, 12(3): 214-221.

GIL MARTínEZ, Alfredo Gil. 2010. Técnicas culinarias. Madrid: Ediciones AKAL.

HeRRERA, P. Javier, Pablo IGLESIAS, Jairo Javier GARCÍA SÁNCHEZ, et al. 2009. «JaDaCook 2: Cooking Over Ontological Knowledge». 8th International Conference on Case-Based Reasoning (ICCBR-09): 279-288.

HIGMAN, Barry. W. 1998. "Cookbooks and Caribbean Cultural Identity: An English-Language Hors D'Oeuvre». NWIG: New West Indian Guide / Nieuwe West-Indische Gids 72 (1-2): 77-95.

INSTITUTO DE INVESTIGACIÓN RAFAEL LAPESA DE LA REAL ACADEMIA ESPAÑOLA. 2013. Corpus del Nuevo diccionario histórico (CDH). Consultado el 6 de febrero de 2018. http://web.frl. es/CNDHE

IRELAND, Jayne D. y A. MøLLER. 2010. «LanguaL Food Description: a Learning Process». European Journal of Clinical Nutrition 64: S44-S48.

JERMSURAWONG, Jermsak y Nizar HABASH. 2015. «Predicting the Structure of Cooking Recipes». Proceedings of the 2015 Conference on Empirical Methods in Natural Language Processing. Lisbon, Portugal: 781-786.

JOHNSTON, Josée y Shyon BAUMANN. 2014. Foodies: Democracy and Distinction in the Gourmet Foodscape. Nueva York y Londres: Routledge. Edición Kindle.

KAMEL BOULOS, Maged N. et al. 2015. «Towards an «Internet of Food»: Food Ontologies for the Internet of Things». Future Internet 7 (4): 372-392.

KARLIN, Robin F. 1988. SEAFACT: Semantic Analysis for Animation of Cooking Tasks. University of Pennsylvania Department of Computer and Information Science.

LARA, Luis Fernando. 1999. "Término y cultura: hacia una teoría del término». En Terminología y modelos culturales, ed. por María Teresa Cabré y Mercè Lorente, Barcelona: Institut Universitari de Lingüística Aplicada, Universitat Pompeu Fabra. Edición Kindle.

MALMAUD, Jon et al. 2014. "Cooking with Semantics". Proceedings of the ACL 2014 Workshop on Semantic Parsing. Baltimore, Maryland USA: 33-38.

MARTínEZ MONZÓ, Javier y Purificación GARCíA SEGOVIA. 2015. Gastronomía, alimentación y nutrición. Madrid: Editorial Síntesis.

MONTANARI, Massimo. 2004. La comida como cultura. Trad. Álvarez, Alfredo. Gijón: Trea.

NAVARRO, Carmen. 2008. "Neología y formación de palabras en el lenguaje de la gastronomía». La comunicación especializada. New York: Peter Lang, 271-292.

OLESCHUK, Merlin. 2017. «Foodies of Color: Authenticity and Exoticism in Omnivorous Food Culture». Cultural Sociology 11 (2): 217-233.

ORTIz CuadRa, Cruz Miguel. 2006. Puerto Rico en la olla, ¿somos aún lo que comimos? Madrid: Doce Calles.

PENNINGTON, Jean A. T. 1994. "LANGUAL: A Food Description Language». Terminology 1 (2): 277-289.

PÉREZ OREJA, Nuria, et al. 2003. Procesos de cocina. Madrid: Síntesis.

Ismarie DIAAZ FLORES

La terminología de la gastronomía puertorriqueña: reflexiones en torno a su trasfondo y representación
CLINA

vol. 4-2, December 2018, 181-205

elSSN: 2444-1961

Ediciones Universidad de Salamanca - CC BY-NC-ND 
PIcó, Fernando. 2008. Historia general de Puerto Rico. 4. ${ }^{2}$ ed. rev. y aum. San Juan: Huracán. Colección Huracán Academia.

SAIYA, James. «Recipe Markup Language (RecipeML)». 2002. Format Data.

SOURCE FORGE. REML: The Recipe Exchange Markup Language. Consultado el 11 de febrero de 2019. http://reml.sourceforge.net/

StEWART TRUSWELL, A. et al. 1991. «INFOODS Guidelines for Describing Foods: A Systematic Approach to Describing Foods to Facilitate International Exchange of Food Composition Data». Journal of Food Composition and Analysis 4(1): 18-38.

TASSE, Dan y Noah A. SMITH. 2008. SOUR CREAM: Toward Semantic Processing of Recipes. Pittsburgh, PA: Carnegie Mellon University.

VIDAL ClARAMONTE, M. ${ }^{a}$ Carmen África y Pamela FABER. "Translation and Food: The Case of Mestizo Writers». Journal of Multicultural Discourses 12 (3):189-204.

ValldeJuli, Carmen Aboy. 2011. Puerto Rican Cookery. 35. ${ }^{2}$ ed. Gretna [La.]: Pelican Pub. Co.

VALLdEJULI, Carmen Aboy. 2011. Cocina criolla. 68. ${ }^{a}$ ed. Gretna [La.]: Pelican Pub. Co.

VIUDAS CAMARASA, Antonio. 1982. «El lenguaje técnico de un recetario de cocina». Anuario de estudios filológicos 5: 219-229. 
